\title{
A portable and affordable extensional rheometer for field testing
}

Bart Hallmark ${ }^{\dagger}$, Matthew Bryan, Ed Bosson, Simon Butler, Tom Hoier, Ole Magens, Nicolas Pistre*, Lee Pratt, Betsy-Ann Ward, Sam Wibberley and D. Ian Wilson

Department of Chemical Engineering and Biotechnology, New Museums Site, Pembroke Street, Cambridge, CB2 3RA, UK

"École Nationale Supérieure de Techniques Avancées, 828 Boulevard des Maréchaux, 91120

Palaiseau, Paris, France.

†Corresponding author: bh206@cam.ac.uk

Draft manuscript

Submitted to Measurement Science and Technology

August 2016

(C) The authors

\section{Abstract}

Extensional shear testing is often needed to characterise the behaviour of complex fluids found in industry and nature. Traditional extensional rheometers are typically expensive, fragile and heavy and are only suited to making measurements in a laboratory environment. For some applications, it is necessary to make in-situ rheological measurements where, for example, fluid properties change rapidly over time or where laboratory facilities are unavailable. This paper reports the development and validation of an inexpensive, lightweight and robust 'open source' extensional rheometer, Seymour II. Validation was carried out experimentally and computationally. Measurements on a Newtonian fluid (492 mPa s Brookfield silicone oil) yielded results of $510 \pm 51 \mathrm{mPa} \mathrm{s}$; these are comfortably within the range of $\pm 10 \%$ which other authors have quoted for extensional techniques using laboratory rheometers. Comparison of the observed filament thinning dynamics to those obtained using computational fluid dynamics (CFD) gave good qualitative agreement. Use of Seymour II at the University of Cambridge Botanic Gardens revealed that the mucilage of the 'Crane flower', Strelitzia reginae, was a viscoelastic fluid whose extensional response could be described by a two-mode Giesekus equation. Engineering drawings and image analysis code for Seymour II are available for download at the project website, http://www.seymourII.org/ 


\section{Introduction}

Many functional liquids found in nature exhibit complex rheological behaviour. For example, carnivorous plants such as pitcher plants rely on the stickiness of the liquid held in their jug-shaped leaves to trap insects to supply the plant with nutrients ${ }^{[1]}$. These biological materials are typically complex fluids - they are often aqueous solutions of long-chain biopolymers, which impart viscoelastic behaviour and, in particular, high extensional viscosity. Rheometers that can characterise the apparent shear viscosity of a complex fluid have been available for many years, however devices that can accurately a complex fluid's extensional response in isolation from shear effects are relatively recent ${ }^{[2]}$.

One technique that has been used extensively over the past two decades is that of capillary breakup rheometry; the basic principle of this technique is shown in Figure 1. A small sample of the test fluid is loaded between two parallel plates, which are both drawn apart rapidly; this causes a liquid filament to form that subsequently reduces in diameter under the action of surface tension and ultimately breaks. Capillary breakup rheometers track the mid-filament diameter as a function of time using optical means: photo cells, laser micrometers and high-speed video imaging have all been used.

The liquid filament microrheometer ${ }^{[3]}$, developed by Bazilevsky and co-workers, was able to use fluid samples as small as $10 \mu 1$ and was capable of interrogating the response of fluids with relaxation times

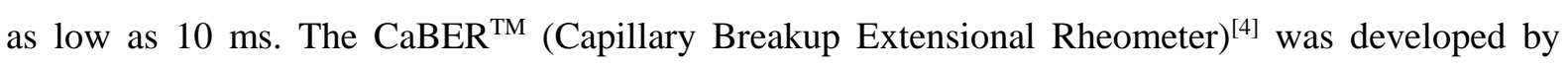
McKinley and co-workers at MIT at the turn of the millenium in conjunction with the Cambridge Polymer Group; in this device laser micrometry is used to track the mid-filament diameter. The $\mathrm{CaBER}^{\mathrm{TM}}$ is currently the only readily-available extensional rheometer on the market. The Cambridge TriMaster $^{\mathrm{TM}[5]}$ was developed by Tuladhar and Mackley at the University of Cambridge in 2008; the construction of this device was motivated by the desire to study low viscosity inkjet liquids and uses high-speed video imaging and subsequent image analysis to obtain data on the mid-filament diameter. The TriMaster ${ }^{\mathrm{TM}}$ is capable of acquiring images roughly every $25 \mu \mathrm{s}^{[6]}$.

Insight into the fluid mechanics of filament stretching has been greatly assisted by computational simulation: different physical phenomena can be uncoupled from one another with relative ease and their effect on the evolution of filament diameter and filament shape can be explored systematically. Numerous authors have examined the filament stretching behaviour of both Newtonian and viscoelastic liquids when subject to increasing extensional strain ${ }^{[7-11]}$ using finite-element based approaches. These authors were able to examine the relative interplay of physical effects such as surface tension, inertia, gravity, viscoelastic stress and strain hardening up to Hencky strains approaching $6^{[11]}$. A variety of viscoelastic models were used in these studies, including FENE-CR ${ }^{[7]}$, Oldroyd-B, linear PTT and Giesekus $^{[8,10,11]}$; an excellent introduction to viscoelasticity and its mathematical description can be found in the book authored by Bird and co-workers ${ }^{[12]}$. 
Simulation of the step strain response of liquid filaments allows direct comparison between theory and experimental measurements from capillary breakup rheometry, enabling differing rheological constitutive equations to be evaluated in terms of their suitability to describe the evolution of the filament shape and any subsequent instabilities that may occur. Commercial finite-element packages, such as Polyflow ${ }^{\mathrm{TM}[13]}$ and COMSOL Multiphysics ${ }^{\mathrm{TM}[14]}$, and bespoke codes using hybrid finite-element and finite-volume approaches ${ }^{[15,16]}$ have all been used to tackle this problem. A range of different rheological constitutive equations have been used by these authors to successfully describe the experimental response of various types of simple and complex fluid, including Newtonian ${ }^{[14]}$, Giesekus $^{[15-17]}$, Oldroyd-B ${ }^{[14-16]}$, PTT $^{[15,16]}$ and FENE-CR ${ }^{[14]}$.

Conventional extensional rheometers, like most laboratory rheometers, are heavy, delicate and expensive. Sometimes it is not possible to test the extensional response of certain fluids in a laboratory: pitcher plant fluids are a good example since their extensional behaviour changes as a function of time, most likely due to the action of enzymes on the sugar polymers in solution ${ }^{[18]}$. There is therefore a need for a portable device for measuring extensional behaviour in the field: it would also be highly desirable for it to function in remote locations (i.e. lightweight, robust, simple utilities) as well as affordable.

This paper reports the development of a lightweight capillary breakup rheometer: Figure 2 illustrates the operating principle. A sample of liquid, between $0.5 \mu 1$ and $1 \mu 1$ in volume, is placed between two pistons and one of the pistons is raised abruptly, promoting the formation of a slender filament of diameter $D$ which becomes thinner over time. Surface tension causes the filament to narrow and this is resisted by the extensional viscosity of the fluid and, if present, the fluid's elasticity. The filament will eventually break, but liquids with significant viscoelasticity may exhibit additional effects, such as 'beads-on-a-string' behaviour ${ }^{[19,20]}$. Simple theory that describes filament thinning for various types of liquid has been reported previously, and Table 1 summarises some results for the evolution of filament diameter, $D$, from initial value, $D_{0}$, over time $t$.

The present device is based on that reported by Collett and co-workers ${ }^{[18]}$ and differs from most capillary breakup rheometers in that only one piston is moved. Advances in digital camera resolution and laptop PC processing power mean that images can be captured and analysed rapidly to give the minimum filament diameter, $D$. The device has only one moving part, the upper piston, making it more robust. The piston is moved by a standard solenoid, which can be actuated by DC battery power: it is therefore readily replaceable as well as lightweight. The main requirement of the solenoid is that the piston movement is rapid; the unit employed here gave a translation time of less than $10 \mathrm{~ms}$. Figure 3 shows a schematic and a photograph of the assembled device. Data are acquired by a laptop with a USB 3 connection, which allows real time transfer of the data as well as powering the camera. The frame can be assembled in minutes and the total mass of the system, without laptop or USB lead, is less than $1 \mathrm{~kg}$. 
The device, known as 'Seymour II', was developed from the 'Seymour' device presented by Collett et al. ${ }^{[18]}$ and incorporated learning gained during field studies of the rheology of the trapping fluid ${ }^{[1]}$ of a particular species of pitcher plant, Nepenthes rafflesiana, undertaken by Dr Ulrike Bauer (Plant Sciences, University of Bristol) in Brunei in 2014. In addition to a new mounting arrangement, the software for capturing and processing images has been rewritten: the design drawings and software code are available as freeware.

\section{Description}

\subsection{Seymour I/ construction}

A schematic diagram of Seymour II along with an annotated photograph can be found in Figure 3. Fabrication drawings are available at [Address to be specified]. The rheometer is designed and built around a battery-powered solenoid that is capable of actuating the top piston: the top piston should have a maximum displacement of about $4 \mathrm{~mm}$. The solenoid used in the current embodiment of Seymour II is a 8.02.13.62 (HE \& BS Benson Ltd., Newmarket, UK), and draws 0.67 A at 6 V DC. An O ring is used to adjust the maximum travel of the top piston such that a filament of the fluid under test firstly forms once the piston has stopped moving, and then breaks in a reasonable timescale. If the piston displacement is too small a fluid filament will not form; with too large a displacement the fluid filament will rupture whilst the piston is still moving. The stationary bottom piston is mounted in the lower body of the rheometer and the solenoid is housed in the upper body. The upper and lower rheometer bodies are connected together by two $8 \mathrm{~mm}$ diameter plain-gauge rods and by one section of studding (M6 $\times$ $165 \mathrm{~mm}$ ): rotation of a tapped knob mounted on the studding and constrained within the upper rheometer body allows vertical adjustment of the initial gap between the pistons prior to piston movement, $l_{0}$.

The rheometer assembly is attached to a section of slotted aluminium profile (30 mm x $60 \mathrm{~mm}$ x 400 $\mathrm{mm}$ ), onto which is also attached a lens support and a torch support. The lens support is sized to grip the lens of choice; in the device used here a Leica Monozoom 7 unit was used, attached to a solid-state high-speed camera (Ximea MQ003MG-CM) capable of up to 500 frames per second at a resolution of $640 \times 480$ pixels. The Ximea camera requires a USB3 connection to a PC equipped with suitable image processing software. For Seymour II, an Intel ${ }^{\circledR}$ Core $i 7^{\circledR}$ laptop was used, running Ubuntu Linux and a bespoke piece of software was written in $\mathrm{C}++$ to extract filament diameter information.

The components for Seymour II are listed in Table 2; for the purposes of fabrication, a full set of engineering drawings and the $\mathrm{C}++$ code for image analysis are available from [Address to be specified].

\subsection{Seymour II experimental protocol}

Prior to performing a capillary breakup rheometry test on Seymour II, the surface tension of the fluid under investigation must be obtained, either experimentally or from literature sources. There are many 
techniques that can be used to measure surface tension experimentally, and a number of good reviews are present in the literature ${ }^{[21,22]}$.

The experimental protocol used to operate Seymour II is given below.

1. Record the laboratory temperature and clean the rheometer pistons thoroughly with isopropanol. Ensure all isopropanol has evaporated prior to loading the test fluid.

2. Assemble the component parts of Seymour II and attach the rheometer body, the lens holder and the torch holder onto the slotted aluminium profile. Attach the camera to the PC and start the image analysis software in 'visualisation mode' such that the pistons can be seen on the PC screen.

3. Use feeler gauges to adjust the initial gap between the two pistons to be $0.6 \mathrm{~mm}$. Adjust the ' $\mathrm{o}$ ' ring such that the final gap allows a filament to form and break; the fluid under test will determine the optimal gap sized, hence some preliminary experimentation will be required to set this.

4. Once the final gap size has been set, and using the image analysis software, ensure that the top and bottom pistons are vertically aligned and in sharp focus.

5. Ensuring that the solenoid is switched off, load a sample of the fluid to be tested into the gap in between the upper and lower piston. Great care should be taken to avoid any of the fluid wetting the vertical surfaces of the pistons; should wetting occur, fluid should be removed carefully with an absorbent medium.

6. Put image analysis software into 'recording' mode with the camera frame rate set at 500 frames per second. Promptly switch on the solenoid to separate the upper and lower pistons, and keep the solenoid activated until filament breakup has occurred, or until the laptop has finished recording the image sequence (whichever happens first). If the fluid filament failed to break, then the $\mathrm{O}$ ring on the upper piston should be adjusted to increase the final gap size.

7. Ensuring that the solenoid is switched off, remove the remainder of any fluid from the rheometer pistons and clean thoroughly with isopropanol.

8. Repeat the experiment a minimum of five $\mathrm{e}^{[4]}$ times and perform data analysis.

\subsection{Seymour II data analysis}

The analysis of extensional rheometry data differs to that of shear rheometry. When analysing the latter, values of the apparent viscosity can be usually be deduced without having to presume a rheological response of the test fluid. This is not the case for extensional testing, where it is necessary to determine which theoretical treatment best fits the experimental filament thinning data. Table 1 lists some of the most commonly-used filament thinning expressions, including the response of a Newtonian fluid ${ }^{[23,24]}$ and commonly used viscoelastic models: upper convected Maxwell(UCM $)^{[24]}$, single mode Giesekus $\operatorname{model}^{[25]}$, FENE $^{[23,26]}$ and FENE-P $\mathrm{P}^{[27]}$. 
Care has to be taken when analysing extensional rheometry data. Firstly, it is necessary to find the region within the recorded data that corresponds to filament thinning under the action of surface tension alone; this may only be part of the data that has been acquired, and sometimes may not be obvious. Then, a selection of different theoretical models can be fitted in turn to an appropriate range of the filament thinning data; the response of the fluid is then deemed to be described by the model whose coefficient of determination is closest to 1 . Sometimes, none of analytical expressions listed in Table 1 may regress sufficiently accurately to the experimental data and more complex models, such as a multimode Giesekus ${ }^{[28]}$, may need testing. Once a good fit with a given model has been obtained, there still may not be enough data to gain a full rheological characterisation of the fluid. For example, it is not possible to obtain a unique set of viscoelastic parameters for the Giesekus model from extensional testing alone, only the ratios of certain model parameters (the mobility parameter and the Giesekus viscosity) can be obtained ${ }^{[28,29]}$. For certain applications, however, the parameters extracted from extensional testing alone can give sufficient information to track fluid property changes as a function of time, or to provide a comparison between fluids from different origins.

The data fitting procedure is illustrated in Figure 4, where three distinct regions are evident: Region (I), where the filament diameter is constant, corresponds to measurements obtained prior to piston movement; Region (II), where a rapid reduction in filament diameter occurs, corresponds to the time when the rheometer pistons are moving and the fluid is being actively stretched; the region where useful measurements can be taken, labelled (III), corresponds to filament thinning under the action of surface tension alone. Any data following the monotonically-decreasing filament thinning regime has been discarded since either the filament will have broken, and only artefacts of the image analysis may be present, or a viscoelastic effect such as beads-on-a-string ${ }^{[19,20]}$ will be occurring, resulting in an unsteady relationship between filament diameter and time.

One of two approaches can then be taken to analyse the data in region (III). For viscoelastic fluids, it is usual to non-dimensionalise the filament diameter with respect to $D_{0}$, the filament diameter at the start of region (III): this is labelled in Figure 4. $D / D_{0}$ is then plotted against time since the start of region III and the viscoelastic filament thinning expressions in Table 1 can then be tested for their respective goodness of fit.

Interestingly, in the absence of viscoelastic effects, the shape of the fluid filament starts to significantly affect the filament thinning process ${ }^{[4]}$; a second approach, therefore, must be used for a Newtonian fluid if values of viscosity are to be compared with those obtained by shear testing. The methodology is discussed in detail by McKinley and Tripathi ${ }^{[4]}$ and stems from the geometric simplification used to derive Newtonian filament thinning expression. The derivation assumes, amongst other things, that the fluid filament is an infinitely-long cylinder, with zero applied axial force and that the filament thinning process is non-inertial. In reality, the shape of the fluid bridge between the two pistons is not cylindrical 
and this results in the presence of a significant axial force during the early stages of filament thinning. The fluid filament is never truly axially uniform, rather that the axial force becomes negligible during the final stages of filament thinning.

Consequently, capillary thinning data for Newtonian fluids is only analysed in the region near filament breakup, region (IV) on Figure 4, where radial forces are significantly larger than axial forces. Additionally, a correction factor, termed the 'shape factor' $(X)$, is applied to the Newtonian filament thinning expression to account for the filament's non-cylindrical shape. The value of $X$ can be computed theoretically, as described in a number of publications ${ }^{[30-32]}$ : for the purposes of making pragmatic viscosity measurements of Newtonian fluids, it has been demonstrated ${ }^{[4]}$ that use of the Papageorgiou solution for the shape factor ${ }^{[30]}, X=0.7127$, is appropriate. In the region near filament breakup, the gradient of the filament thinning profile can be now be compared to theory viz:

$$
\frac{\Delta D}{\Delta t}=-\frac{(2 X-1) \alpha}{3 \eta_{0}}
$$

or

$$
D(t)=\frac{0.1418 \alpha}{\eta_{0}}\left(t_{c}-t\right)
$$

Here, $\alpha$ is the surface tension, $\eta_{0}$ is the Newtonian viscosity and $t_{c}$ is the time at capillary breakup. The solid grey line in Figure 4 illustrates how Equation (2) can be fitted to experimental data; the dashed grey line in this Figure illustrates the difference in gradient at earlier times, when axial forces are nonnegligible.

\subsection{Seymour II validation}

Validation of Seymour II was carried out using a combined experimental and theoretical approach. Firstly, a series of measurements of piston position as a function of time were taken such that the piston movement could be characterised and the piston velocity measured as a function of time. An ideal extensional rheometer should be able to apply a step change in position to the pistons in order to form a fluid filament; pragmatically, a fast, monotonic, piston movement is required. Next, a series of ten, identical, filament thinning experiments were carried out using a Newtonian viscosity standard (Brookfield silicone oil, $492 \mathrm{mPa} \mathrm{s}$ ). Some surface tension data appear in the literature for Brookfield silicone oil viscosity standards: Keller and co-workers ${ }^{[33]}$ quote a $19.7 \mathrm{mN} / \mathrm{m}$ and $20.9 \mathrm{mN} / \mathrm{m}$ for 50 $\mathrm{mPa}$ s and $1000 \mathrm{mPa}$ s Brookfield viscosity standards respectively, and Starov and co-workers ${ }^{[34]}$ report a value of $22.5 \mathrm{mN} / \mathrm{m}$ for a viscosity standard of $91 \mathrm{mPa}$. Furthermore, Schweizer and Kister ${ }^{[35]}$ quote 
a surface tension value of $21.7 \mathrm{mN} / \mathrm{m}$ for a Brookfield silicone oil of viscosity $98.8 \mathrm{~Pa}$ s: a representative surface tension value of $21.0 \mathrm{mN} / \mathrm{m}$ was used here. The density was $970 \mathrm{~kg} / \mathrm{m}^{3[36]}$. The initial gap between the pistons was $0.6 \mathrm{~mm}$ and the final gap was set to $1.4 \mathrm{~mm}$. The symmetry of the fluid filament was checked in each of the data sets - an experiment was discarded if symmetry was broken. The data analysis method for a Newtonian fluid, outlined in the previous section, was then used to extract a value for the fluid viscosity for each experiment.

The theoretical performance of Seymour II was then computed using a commercial CFD package, Polyflow $^{\mathrm{TM}}$ (Ansys Inc.). A two-dimensional, axisymmetric, problem was defined on an initially square domain, with side length $0.6 \mathrm{~mm}$ (since Seymour II had an initial gap and $0.6 \mathrm{~mm}$, and used pistons 1.2 $\mathrm{mm}$ in diameter); the domain was meshed with 6560 triangular elements. Boundary conditions of symmetry and zero tangential and normal velocities were applied to the left-hand and lower edge of the domain respectively. A free surface boundary condition was defined on the right-hand edge of the domain, with its location pinned at its intersection with the upper and lower edges; surface tension was specified to act on this boundary with a value of $21 \mathrm{mN} / \mathrm{m}$. The upper edge of the domain was set to have a translational velocity equal to that experimentally-measured for the top piston of Seymour II, decaying exponentially and rapidly to zero once the motion had ceased. The upper edge's translational velocity was coded as a user defined function in the rule-based language CLIPS $^{[37]}$; this can then be used by Polyflow ${ }^{\mathrm{TM}}$.

The material properties of viscosity and density were set at $0.492 \mathrm{~Pa} \mathrm{~s}$ and $970 \mathrm{~kg} / \mathrm{m}^{3}$ respectively, inertia was included in the calculation, a Langrangian remeshing algorithm was specified on the domain and adaptive mesh refinement enabled with the program's default settings. The transient solver was used with an upper time limit of $105 \mathrm{~ms}$, an initial time step of $0.2 \mathrm{~ms}$, a minimum time step of $0.1 \mathrm{~ms}$ and a maximum time step of $1 \mathrm{~ms}$. The simulation was run in approximately ten minutes on 15 cores of a dual CPU Intel Xeon E5-2630 server, with a clock speed of $2.6 \mathrm{GHz}$. An example of an initial and a deformed mesh along with the CLIPS code is given in the supplementary material that accompanies this paper.

Once converged, the computed profile of $D / D_{0}$ as a function of time could then be extracted from the results and compared to those profiles that had been obtained experimentally. Moreover, the computed shape of the fluid filament at a number of times during the filament thinning process could be obtained, and compared to the experimentally-observed fluid filament. This allows the data obtained from Seymour II to be compared to an ideal data set, hence validating the accuracy of the experimental protocol and of the image acquisition and analysis.

The final part of the validation exercise was to demonstrate the portability of Seymour II and the ability to 'take the laboratory to the sample' rather than vice versa. Seymour II was taken to the University of Cambridge Botanic Gardens to investigate the properties of mucilage extracted from the 'Crane 
Flower', Strelitzia reginae. For these tests, mucilage was extracted from open flowers of S. reginae using a micropipette and transferred to Seymour II; an initial piston gap of $0.6 \mathrm{~mm}$ and a final gap of $1.4 \mathrm{~mm}$ was used. A value for the surface tension of $S$. reginae mucilage was not available, hence the value for water was used. Detailed analysis of the physical properties of $S$. reginae mucilage would be required if these preliminary studies were to be extended.

\section{Results and discussion}

\subsection{Seymour II piston movement}

A plot of piston displacement as a function of time for ten different tests is shown in Figure 5. Good reproducibility can be seen across the series of tests, and it can be seen that the time taken for piston displacement is between $8.5 \mathrm{~ms}$ and $9 \mathrm{~ms}$. This is roughly 7 times slower than the TriMaster ${ }^{\mathrm{TM}}$ when configured at its fastest piston velocity of $500 \mathrm{~mm} \mathrm{~s}^{-1[38]}$, but similar to the system response time of the $\mathrm{CaBER}^{\mathrm{TM}[39]}$.

A plot of average piston velocity for Seymour II is shown in Figure 6; these data were obtained using centred differencing on the average piston displacement. It can be seen that the piston velocity accelerates over the course of its motion to $450 \mathrm{~mm} \mathrm{~s}^{-1}$, which represents $90 \%$ of the maximum speed of the TriMaster. Also shown on the Figure are two functions that were fitted to the accelerating and decelerating sections of the piston motion for the purposes of numerical modelling. Over the range $0<$ $t<0.0086 \mathrm{~s}$, where the piston accelerates, the piston velocity, $v_{\mathrm{p}}$, is described by the cubic polynomial:

$v_{p}=3.34662 \times 10^{-3}-8.43158 \times 10^{-3} t+1.14724 \times 10^{-2} t^{2}-5.11266 \times 10^{-4} t^{3} \quad 0<t<0.0086 \mathrm{~s}$

Specifying the piston velocity to instantaneously fall to zero for $t \geq 0.0086 \mathrm{~s}$ caused the numerical simulation to diverge, hence the deceleration of the piston was modelled as a rapid exponential decay to (essentially) rest, viz:

$$
v_{p}=0.4541399 \exp (-5(t-0.0086)) \quad t \geq 0.0086 \mathrm{~s}
$$

\subsection{Filament thinning of silicone oil}

Ten experiments on Seymour II were carried out. Three of these experiments (A, B and C) either exhibited significant filament asymmetry, due to the silicone oil having partially wetted the vertical sides of the lower piston, or showed that the lower liquid pool drained visibly during filament thinning: these results were discarded. Numerical simulation of the filament thinning of silicone oil on Seymour 
II using Polyflow ${ }^{\mathrm{TM}}$ took $656 \mathrm{~s}$ on 15 cores (equivalent to 7003 seconds of single core calculation) and used 4196 MB of RAM.

The sequence of images shown in Figure 7 compare the experimentally-observed shape of the silicone oil filament, illustrated in the right-hand half of each image, to the numerically-calculated filament shape, illustrated in the left-hand half of each image, for a selection of time intervals between filament formation and filament break-up. There is strong visual similarity between the experimental and numerical results in this Figure, demonstrating that the mechanical operation of Seymour II results in the formation of a fluid filament whose behaviour is consistent with established theory.

The plot shown in Figure 8 shows the quantitative similarity between the simulation and the experiments on Seymour II. There are a cluster of silicone oil filament thinning profiles measured using Seymour II (experiments D, F, G, I and J) that lie close to, or on, the profile predicted by simulation; the data from experiments $\mathrm{E}$ and $\mathrm{H}$ lie a little way away from the predicted values, which is attributed to slight differences in the initial gap between the rheometer pistons.

Application of the analysis for a Newtonian fluid outlined in the previous section yields the viscosity values shown in Table 3 . The error values were calculated by assuming that time was accurate to $\pm 1 \mathrm{~ms}$ at a frame rate of 500 frames per second; that diameters were accurate to within \pm 1 pixel, at $5.8 \mu \mathrm{m}$ per pixel; and that the surface tension value used was within $\pm 1 \mathrm{mN} / \mathrm{m}$ of that of the experimental fluid. The average viscosity resulting from seven experiments is $510 \pm 51 \mathrm{mPa} \mathrm{s}$, which is within $4 \%$ of the quoted standard value of $492 \mathrm{mPa}$ s, which itself is subject to a $\pm 2 \%$ uncertainty ${ }^{[40]}$. Previous work on using extensional rheometry to extract Newtonian viscosity values has attained a reproducibility of $\pm 10 \%{ }^{[4]}$, hence the results reported here for Seymour II are well within the expected accuracy of this technique.

\subsection{Extensional behaviour of S. reginae mucilage}

Preliminary filament thinning data for two samples of S. reginae mucilage, measured using Seymour II in situ at the University of Cambridge Botanic Gardens, are shown in Figure 9.The plots indicate that S. reginae mucilage is not a Newtonian fluid. Filament thinning curves of this nature are typical for viscoelastic polymer solutions where either the solvent viscosity dominates the initial filament dynamics $^{[23,27,41,14]}$ or where there are two viscoelastic contributions with significantly different time constants $^{[28,11,13]}$. Recent work ${ }^{[28]}$ has derived an ordinary differential equation (ODE), Equation (5), that can be fitted to filament thinning curves of the form shown in Figure 9 to extract parameters for a twomode Giesekus model ${ }^{[42]}$. The ODE requires numerical solution in, for example, Microsoft Excel ${ }^{\mathrm{TM}}$ or MATLAB ${ }^{\mathrm{TM}}$; full details of the solution algorithm, and of the assumptions that underlie the analysis, are contained within the paper by Hallmark and co-workers ${ }^{[28]}$. 


$$
\frac{d D}{d t} \approx-\frac{D^{2}\left(\lambda_{1} \tau_{z z, 2}+\lambda_{2}\left(\tau_{z z, 1}+\frac{2 \lambda_{1} \tau_{z z, 1}^{2}}{\eta_{E, 1}}+\frac{2 \lambda_{1} \tau_{z z, 2}^{2}}{\eta_{E, 2}}\right)\right)}{6 \alpha \lambda_{1} \lambda_{2}}
$$

The loci of points shown in Figure 9 are the best fit of Equation (5) to the experimental data, with the constraint that fitting should accurately capture the filament thinning profile in the lead up to filament rupture. The coefficient of determination, $R^{2}$, is 0.87 and 0.82 for samples A and B respectively; the initial region of filament thinning is captured accurately in each case, with the later stages of filament thinning showing some deviation between theory and experimental data. Values of the Giesekus extensional viscosity ${ }^{[29]}$ and relaxation times for the short and long modes are given in Table 4. The long mode relaxation time is approximately five times higher than that for the short mode, with values ranging between $0.60 \mathrm{~s}$ and $0.65 \mathrm{~s}$ for the short mode and between 3.3 and $3.5 \mathrm{~s}$ for the long mode. The absolute values of the Giesekus extensional viscosity show a greater degree of inter-sample variation, but typically with long mode values an order of magnitude higher than short mode values; the range is $49 \mathrm{~Pa} \mathrm{~s}$ and $120 \mathrm{~Pa}$ s for the short mode and between $890 \mathrm{~Pa}$ and $1350 \mathrm{~Pa}$ s for the long mode. These data illustrate that the rheological response of $S$. reginae mucilage follows that typical for a multi-mode polymer solution.

\section{Conclusions}

This paper has reported the construction, experimental protocol and data analysis techniques for an 'open source', inexpensive and lightweight extensional rheometer, Seymour II. Validation of the rheometer has been carried out using a Newtonian viscosity standard (492 $\mathrm{mPa}$ s Brookfield silicone oil); results of $510 \pm 51 \mathrm{mPa}$ s were obtained, which are comfortably within the range of $\pm 10 \%$ which other authors have quoted for extensional techniques ${ }^{[4]}$. Visual comparison of the filament dynamics observed using Seymour II to those computed using a commercial CFD package (Polyflow ${ }^{\mathrm{TM}}$ ) show good qualitative agreement.

We also report preliminary rheological data for mucilage from the 'Crane flower', Strelitzia reginae. The mucilage exhibited significant levels of viscoelasticity, which could described in terms of the extensional response of a two-mode Giesekus model. The authors believe these data to be the first quantitative viscoelastic measurements for $S$. reginae mucilage. Short mode relaxation times ranged between 0.60 and $0.65 \mathrm{~s}$ with long mode relaxation times ranging between 3.3 and $3.5 \mathrm{~s}$. Giesekus extensional viscosities ranged between $49 \mathrm{Pas}$ and $120 \mathrm{~Pa}$ s for the short mode and between $890 \mathrm{~Pa} \mathrm{~s}$ and $1350 \mathrm{~Pa}$ s for the long mode. 


\section{Acknowledgements}

Helpful conversations with Dr Ulrike Bauer (Plant Sciences, University of Bristol) are gratefully acknowledged, as is support for a summer internship for NP from ENSTA, a PhD studentship for MPB from Sandvik Hyperion and Ceratizit, and a PhD studentship for OMM from Chemours. We are also grateful to Alex Summers for supporting tests at the University of Cambridge Botanic Gardens. 


\section{References}

1. Bauer U, Grafe TU, Federle W. (2011). Evidence for alternative trapping strategies in two forms of the pitcher plant, Nepenthes rafflesiana. J. Exp. Bot. 62:10, 3683-92.

2. Galindo-Rosales FJ, Alves MA, Oliveira MSN. (2012). Microdevices for extensional rheometry of low viscosity elastic liquids: a review. Microfluid. Nanofluidics. 14:1-2, 1-19.

3. Bazilevsky AV, Entov VM, Rozhkov AN. (1990). In: Oliver DR, editor. Third European Rheology Conference and Golden Jubilee Meeting of the British Society of Rheology. (Dordrecht: Springer Netherlands).

4. McKinley GH, Tripathi A. (2000). How to extract the Newtonian viscosity from capillary breakup measurements in a filament rheometer. J. Rheol. American Institute of Physics) 44:3, 653.

5. Tuladhar TR, Mackley MR. (2008). Filament stretching rheometry and break-up behaviour of low viscosity polymer solutions and inkjet fluids. J. non-Newton. Fluid Mech. 148:1-3, 97-108.

6. Vadillo DC, Hoath SD, Hsiao W-K, Mackley MR. (2011). Proceedings of the 27th international conference on digital printing technologies, NIP27. (Minneapolis, MN, USA: .

7. Sizaire R, Legat V. (1997). Finite element simulation of a filament stretching extensional rheometer. J. non-Newton. Fluid Mech. 71:1-2, 89-107.

8. Yao M, McKinley GH. (1998). Numerical simulation of extensional deformations of viscoelastic liquid bridges in filament stretching devices. J. non-Newton. Fluid Mech. 74:1, 4788.

9. Chandio MS, Matallah H, Webster MF. (2003). Numerical simulation of viscous filament stretching flows. Int. J. Numer. Methods Heat Fluid Flow. 13:7, 899-930.

10. Matallah H, Banaai MJ, Sujatha KS, Webster MF. (2006). Modelling filament stretching flows with strain-hardening models and sub-cell approximations. J. non-Newton. Fluid Mech. 134:1$3,77-104$.

11. Matallah H, Sujatha KS, Banaai MJ, Webster MF. (2007). Single and multi-mode modelling for filament stretching flows. J. non-Newton. Fluid Mech. 146:1-3, 92-113.

12. Bird RB, Armstrong RC, Hassager O. (1987). Dynamics of polymeric liquids. Volume 1 - Fluid Mechanics. (New York: John Wiley and Sons Inc.).

13. Yao M, McKinley GH, Debbaut B. (1998). Extensional deformation, stress relaxation and necking failure of viscoelastic filaments. J. non-Newton. Fluid Mech. 79:2-3, 469-501. 
14. Tembley M, Vadillo D, Mackley MR, Soucemarianadin A. (2012). The matching of a "onedimensional" numerical simulation and experiment results for low viscosity Newtonian and nonNewtonian fluids during fast filament stretching and subsequent break-up. J. Rheol. The Society of Rheology) 56:1, 159.

15. Sujatha KS, Matallah H, Banaai MJ, Webster MF. (2008). Modeling step-strain filamentstretching (CaBER-type) using ALE techniques. J. non-Newton. Fluid Mech. 148:1, 109-21.

16. Webster MF, Matallah H, Sujatha KS, Banaai MJ. (2008). Numerical modelling of step-strain for stretched filaments. J. non-Newton. Fluid Mech. 151:1, 38-58.

17. Yao M, McKinley GH, Debbaut B. (1998). Extensional deformation, stress relaxation and necking failure of viscoelastic filaments. J. non-Newton. Fluid Mech. 79:2-3, 469-501.

18. Collett C, Ardron A, Bauer U, Chapman G, Chaudan E, Hallmark B, Pratt L, Torres-Perez M, Wilson D. (2015). A portable extensional rheometer for measuring the viscoelasticity of pitcher plant and other sticky liquids in the field. Plant Methods. 11:1, 16.

19. Entov VM, Yarin AL. (1984). Influence of elastic stresses on the capillary breakup of jets of dilute polymer solutions. Fluid Dyn. 19:1, 21-9.

20. Clasen C, Eggers J, Fontelos MA, Li J, McKinley GH. (2006). The beads on a string structure of viscoelastic threads. J. Fluid Mech. 556, 283-308.

21. Lee B-B, Ravindra P, Chan E-S. (2008). A critical review: surface and interfacial tension measurement by the drop weight method. Chem. Eng. Commun. Taylor \& Francis Group) 195:8, 889-924.

22. Yuan Y, Lee TR. (2013). In: Bracco G, Holst B, editors. Surface Science Techniques. (Berlin: Springer Verlag).

23. Entov VM, Hinch EJ. (1997). Effect of a spectrum of relaxation times on the capillary thinning of a filament of elastic liquid. J. non-Newton. Fluid Mech. 72:1, 31-53.

24. Stelter M, Brenn G, Yarin AL, Singh RP, Durst F. (2000). Validation and application of a novel elongational device for polymer solutions. J. Rheol. 44, 595.

25. Torres MD, Hallmark B, Wilson DI, Hilliou L. (2014). Natural Giesekus fluids: Shear and extensional behavior of food gum solutions in the semidilute regime. AIChE J. 60:11, 3902-15.

26. Anna SL, McKinley GH. (2001). Elasto-capillary thinning and breakup of model elastic liquids. J. Rheol. 45:1, 115.

27. Wagner C, Bourouiba L, McKinley GH. (2015). An analytic solution for capillary thinning and 
breakup of FENE-P fluids. J. non-Newton. Fluid Mech. 218, 53-61.

28. Hallmark B, Pistre N, Wilson DI. (2016). Characterisation of extensional rheological filament stretching with a dual-mode Giesekus model. AIChE J. , n/a - n/a.

29. Larson RG. (1988). Constitutive Equations for Polymer Melts and Solutions: Butterworths Series in Chemical Engineering. Elsevier Science).

30. Papageorgiou DT. (1995). On the breakup of viscous liquid threads. Phys. Fluids. AIP Publishing) 7:7, 1529.

31. Eggers J. (1997). Nonlinear dynamics and breakup of free-surface flows. Rev. Mod. Phys. American Physical Society) 69:3, 865-930.

32. Brenner MP, Lister JR, Stone HA. (1996). Pinching threads, singularities and the number 0.0304... Phys. Fluids. AIP Publishing) 8:11, 2827.

33. Keller AA, Broje V, Setty K. (2007). Effect of advancing velocity and fluid viscosity on the dynamic contact angle of petroleum hydrocarbons. J. Pet. Sci. Eng. 58:1-2, 201-6.

34. Starov VM, Velarde MG, Radke CJ. (2007). Wetting and Spreading Dynamics. CRC Press).

35. Schweizer PM, Kistler SF. (2012). Liquid Film Coating: Scientific principles and their technological implications. Springer Science \& Business Media).

36. Brookfield. (2010). Material safety data sheet: Fluid 5 to 100,000 cP.

37. Culbert C, Riley G, Dantes B. (2015). CLIPS reference manual. Volume 1: basic programming guide. http://clipsrules.sourceforge.net/documentation/v630/bpg.pdf.

38. Vadillo DC, Tuladhar TR, Mulji AC, Jung S, Hoath SD, Mackley MR. (2010). Evaluation of the inkjet fluid's performance using the "Cambridge Trimaster" filament stretch and break-up device. J. Rheol. American Institute of Physics) 54:2, 261.

39. Thermo-Haake. (2002). CaBER 1 - Quantifying the extensional properties of fluids. http://www.campoly.com/files/8113/5216/6124/CaBER.pdf.

40. Brookfield.

(2010).

Viscosity

Standards. http://www.brookfieldengineering.com/download/files/ViscStdBro.pdf.

41. Fontelos MA, Li J. (2004). On the evolution and rupture of filaments in Giesekus and FENE models. J. non-Newton. Fluid Mech. 118:1, 1-16.

42. Giesekus H. (1982). A simple constitutive equation for polymer fluids based on the concept of deformation-dependent tensorial mobility. J. non-Newton. Fluid Mech. 11:1-2, 69-109. 
43. Liang RF, Mackley MR. (1994). Rheological characterization of the time and strain dependence for polyisobutylene solutions. J. non-Newton. Fluid Mech. 52:3, 387-405. 


\section{List of symbols}

\section{Roman letters}

$\begin{array}{llll}a & - & \text { Giesekus mobility parameter } & (-) \\ b & - & \text { Finite extensibility parameter } & (-) \\ D & - & \text { Filament diameter } & (\mathrm{m}) \\ D_{0} & - & \text { Initial filament diameter } & (\mathrm{m}) \\ D_{p} & - & \text { Plate diameter } & (\mathrm{m}) \\ E_{c} & - & \text { Elasto-capillary number } & (-) \\ g & - & \text { Elastic modulus } & (\mathrm{Pa}) \\ l_{0} & - & \text { Initial gap dimension } & (\mathrm{m}) \\ t & - & \text { Time } & (\mathrm{s}) \\ t_{c} & - & \text { Filament breakup time } & (\mathrm{s}) \\ t^{\prime} & - & \text { Dimensionless time } & (-) \\ v_{p} & - & \text { Piston velocity } & \left(\mathrm{m} \mathrm{s} \mathrm{s}^{-1}\right) \\ X & - & \text { Shape factor } & (-)\end{array}$

\section{Greek letters}

$\begin{array}{llll}\alpha & - & \text { Surface tension } & \left(\mathrm{N} \mathrm{m}^{-1}\right) \\ \eta_{0} & - & \text { Zero shear rate viscosity } & (\mathrm{Pa} \mathrm{s}) \\ \eta_{\mathrm{E}} & - & \text { Extensional viscosity } & (\mathrm{Pa} \mathrm{s}) \\ \lambda & - & \text { Relaxation time } & (\mathrm{s}) \\ \xi & - & \text { Dimensionless diameter } & (-) \\ \tau_{\mathrm{zz}} & - & \text { Axial extra stress } & (\mathrm{Pa})\end{array}$

\section{Subscripts}

$i \quad-\quad i^{\text {th }}$ relaxation mode 


\section{List of tables}

Table 1. A selection of analytical expressions used to describe non-dimensional filament thinning as a function of time

\begin{tabular}{|c|c|c|c|}
\hline $\begin{array}{l}\text { Constitutive } \\
\text { relationship }\end{array}$ & Equation & Comments & Reference \\
\hline Newtonian & $\frac{D}{D_{0}}=1-\frac{(2 X-1) \alpha}{3 \eta_{0} D_{0}} t$ & $\begin{array}{l}X, \text { the shape factor, corrects for } \\
\text { non-cylindrical filaments }{ }^{[4,43]} \text {. }\end{array}$ & $\begin{array}{c}\text { Entov and Hinch }{ }^{[23]} \text {, Stelter } \\
\text { et al. } .^{[24]}\end{array}$ \\
\hline $\begin{array}{l}\text { Upper Convected } \\
\text { Maxwell (UCM) }\end{array}$ & $\frac{D}{D_{0}}=\exp \left(\frac{-t}{3 \lambda}\right)$ & $\begin{array}{l}\text { Allows quantification of } \\
\text { relaxation time. }\end{array}$ & Stelter et al. ${ }^{[24]}$ \\
\hline Giesekus & $(4 a-3) \ln \left(\frac{D / D_{0}+2 \alpha \lambda a / \eta_{0} D_{0}}{1+2 \alpha \lambda a / \eta_{0} D_{0}}\right)-\frac{2 \eta_{0} D_{0}}{\alpha \lambda}\left(D / D_{0}-1\right)=\frac{t}{\lambda}$ & Explicit in $t, \operatorname{not} D$ & Torres et al..$^{[25]}$ \\
\hline FENE & $\frac{D}{D_{0}}=\left(\sum_{i}\left(\frac{g_{i} D_{0}}{2 \alpha}\right) \exp \left(\frac{-t}{\lambda_{i}}\right)\right)^{1 / 3}$ & $\begin{array}{l}\text { Valid for dilute suspensions of } \\
\text { non-interacting FENE } \\
\text { dumbbells. Model has } i \\
\text { relaxation times. }\end{array}$ & $\begin{array}{c}\text { Entov and Hinch }{ }^{[23]} \text {, Anna } \\
\text { and McKinley }\end{array}$ \\
\hline FENE-P & $\begin{array}{c}\left(\frac{1}{1+E_{c}(b+3)}-\frac{1}{1+\xi E_{c}(b+3)}\right)+3 \ln \left(\frac{1+\xi E_{c}(b+3)}{1+E_{c}(b+3)}\right)+4 E_{c} \frac{(b+3)}{(b+2)}(\xi-1) \\
=-\frac{(b+3)^{2}}{b(b+2)} t^{\prime} \\
\xi=\frac{D}{D_{0}} ; t^{\prime}=\frac{t}{\lambda} ; E_{c}=\frac{g D_{0}}{2 \alpha}\end{array}$ & & Wagner et al. ${ }^{[27]}$ \\
\hline
\end{tabular}


Table 2. List of components required to build Seymour II

\begin{tabular}{|c|c|c|c|c|}
\hline Item & Specification & Source & Comment & Price (2016) \\
\hline Laptop & $\begin{array}{l}\text { Core } 17 \text { or equivalent } \\
\text { USB } 3 \text { connection } \\
\text { Ubuntu Linux }\end{array}$ & Hewlett Packard & $\begin{array}{l}\text { A standard laptop with a } \\
\text { USB } 3 \text { drive }\end{array}$ & c. $£ 1000$ \\
\hline Software & $\begin{array}{l}\text { OpenCV v2.4 } \\
\text { Ximea API } \\
\text { gcc or other C++ compiler } \\
\text { cmake } \\
\text { Code supplied with this } \\
\text { project }\end{array}$ & & $\begin{array}{l}\text { Required to build image } \\
\text { processing software - see } \\
\text { http://www.seymourII.org } \\
\text { for more details }\end{array}$ & - \\
\hline Cable & $\begin{array}{l}\text { USB } 3 \text { connection cable, } 1 \\
\text { m long }\end{array}$ & & & c. $£ 20$ \\
\hline Camera & $\begin{array}{l}\text { Digital camera } \\
\text { USB } 3 \text { connection } \\
500 \mathrm{fps} \text {, images } 648-488 \\
\text { pixels }\end{array}$ & $\begin{array}{l}\text { Ximea } \\
\text { MQ003MG-CM }\end{array}$ & & c. $£ 1000$ \\
\hline Lens & $\begin{array}{l}\text { Parfocal } \times 25 \text { microscope } \\
\text { lens }\end{array}$ & $\begin{array}{l}\text { Leica } \\
\text { Monozoom } 7\end{array}$ & Discontinued & $\begin{array}{l}\text { Second hand, c. } \\
£ 200\end{array}$ \\
\hline Light source & White LED torch & & & c. $£ 10$ \\
\hline Solenoid & $\begin{array}{l}\text { Rod diameter around } 1.2 \\
\mathrm{~mm} \text {. } \\
\text { Rod travel up to } 4 \mathrm{~mm} \\
\text { Rod design as per } \\
\text { drawings }\end{array}$ & $\begin{array}{l}\text { Model } \\
8.02 .13 .62, \quad \mathrm{HE} \\
\& \quad \text { BS Benson } \\
\text { Ltd, Newmarket, } \\
\text { UK }\end{array}$ & $\begin{array}{l}\text { Rod geometry will depend } \\
\text { on resolution of camera } \\
\text { and volume of sample. } \\
\text { Power draw } 4 \mathrm{~W} \text { at } 6 \mathrm{~V} \\
\text { DC, duty cycle rating of } \\
100 \% \text {. }\end{array}$ & c. $£ 20$ \\
\hline $\begin{array}{l}\text { Feeler } \\
\text { gauges }\end{array}$ & & & $\begin{array}{l}\text { Metric preferred, } 100 \\
\text { micron to } 1 \mathrm{~mm}\end{array}$ & - \\
\hline O-ring & To fit rod & & & - \\
\hline Framework & & & As per drawings & $\begin{array}{l}\text { Materials } \\
£ 100\end{array}$ \\
\hline
\end{tabular}


Table 3. Viscosity values for $492 \mathrm{mPa}$ s Brookfield silicone oil assuming surface tension of $21 \mathrm{mN} \mathrm{m}^{-1}$

\begin{tabular}{|c|c|}
\hline Experiment & $\begin{array}{c}\text { Viscosity } \\
(\mathbf{m P a} \text { s) }\end{array}$ \\
\hline D & $499 \pm 42$ \\
\hline E & $531 \pm 59$ \\
\hline F & $505 \pm 48$ \\
\hline G & $518 \pm 57$ \\
\hline H & $494 \pm 46$ \\
\hline I & $507 \pm 47$ \\
\hline J & $520 \pm 60$ \\
\hline Average & $\mathbf{5 1 0} \pm \mathbf{5 1}$ \\
\hline Standard deviation & $\mathbf{1 3}$ \\
\hline
\end{tabular}


Table 4. Parameters for Equation (5) for two samples of mucilage from $S$. reginae. Estimated surface tension $72 \mathrm{mN} \mathrm{m}^{-1}$.

\begin{tabular}{|c|c|c|c|c|c|}
\hline \multirow{2}{*}{ Parameter } & \multicolumn{2}{|c|}{ Short mode (1) } & \multicolumn{2}{c|}{ Long mode (2) } & \multirow{2}{*}{ Units } \\
\cline { 2 - 5 } & Sample A & Sample B & Sample A & Sample B & \\
\hline $\boldsymbol{\eta}_{\boldsymbol{E}}$ & 120 & 49 & 1350 & 890 & $\mathrm{~Pa} \mathrm{~s}$ \\
\hline $\boldsymbol{\lambda}$ & 0.65 & 0.6 & 3.5 & 3.3 & $\mathrm{~s}$ \\
\hline
\end{tabular}




\section{Figure captions}

Figure 1. Schematic diagram of conventional capillary break-up rheometry. A sample is loaded between two pistons (a). The pistons move equal distances apart (b), forming a filament with initial mid-point diameter $D_{0}(\mathrm{c})$. The filament then thins under the action of surface tension (d).

Figure 2. Schematic diagram of the operation of Seymour II. A sample is loaded between two pistons (a). The top piston then moves away from the bottom piston $(b$,$) forming a filament with initial mid-$ point diameter $D_{0}$ (c). The filament then thins under the action of surface tension (d).

Figure 3. Seymour II (A) Schematic diagram of mounting and mechanism; (B) photograph

Figure 4. Plot of raw data obtained from Seymour II for a Newtonian fluid (silicone oil). The plot shows a region where the pistons are in their initial position (I), a region where the pistons are moving and a filament of fluid is forming (II) and a region where filament thinning is taking place (III). Also marked is the region where accurate measurement of the Newtonian viscosity can be made ${ }^{[4,30]}$ (IV).

Figure 5. Evolution of piston displacement for ten tests on Seymour II.

Figure 6. Average piston velocity calculated for data sets in Figure 5 Loci show fitted functions: solid line - Equation (1)

Figure 7. Comparison of experimental and Polyflow ${ }^{\mathrm{TM}}$ simulation results for a typical filament thinning experiment on Seymour II using $496 \mathrm{mPa}$ s silicone oil. $t$ is time elapsed since pistons stop moving.

Figure 8. Non-dimensional filament diameter as a function of time for $496 \mathrm{mPa}$ s silicone oil. Points show experimental data collected with Seymour II; locus shows numerical simulation using Polyflow ${ }^{\mathrm{TM}}$. 


\section{List of Figures}

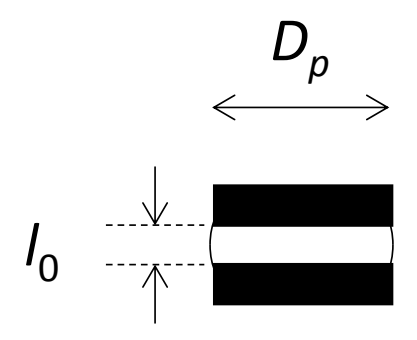

(a)

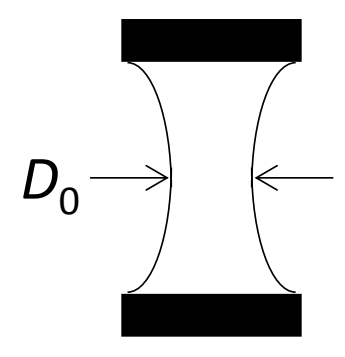

(c)

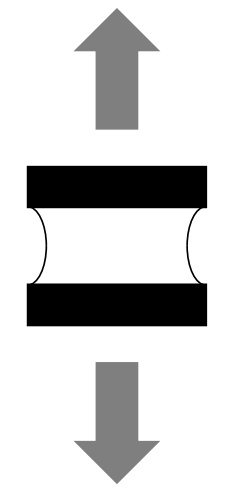

(b)

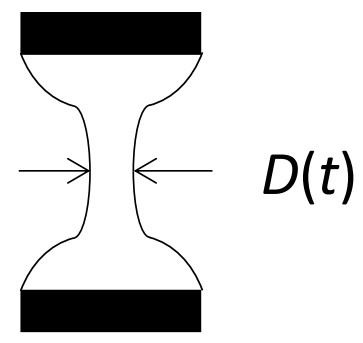

(d)

Figure 1. Schematic diagram of conventional capillary break-up rheometry. A sample is loaded between two pistons (a). The pistons move equal distances apart (b), forming a filament with initial mid-point diameter $D_{0}(c)$. The filament then thins under the action of surface tension (d). 


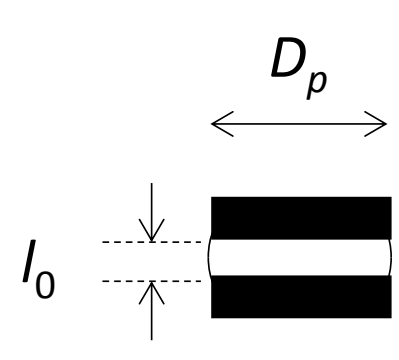

(a)

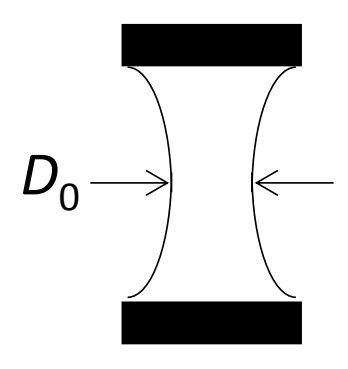

(c)

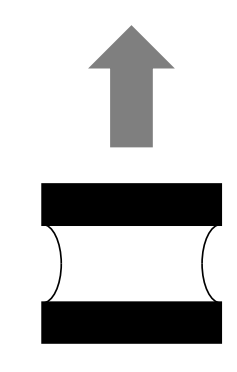

(b)

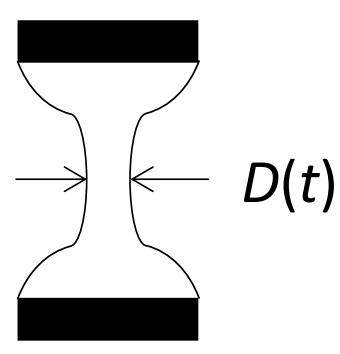

(d)

Figure 2. Schematic diagram of the operation of Seymour II. A sample is loaded between two pistons (a). The top piston then moves away from the bottom piston (b,) forming a filament with initial mid-point diameter $D_{0}(c)$. The filament then thins under the action of surface tension (d). 
(A)

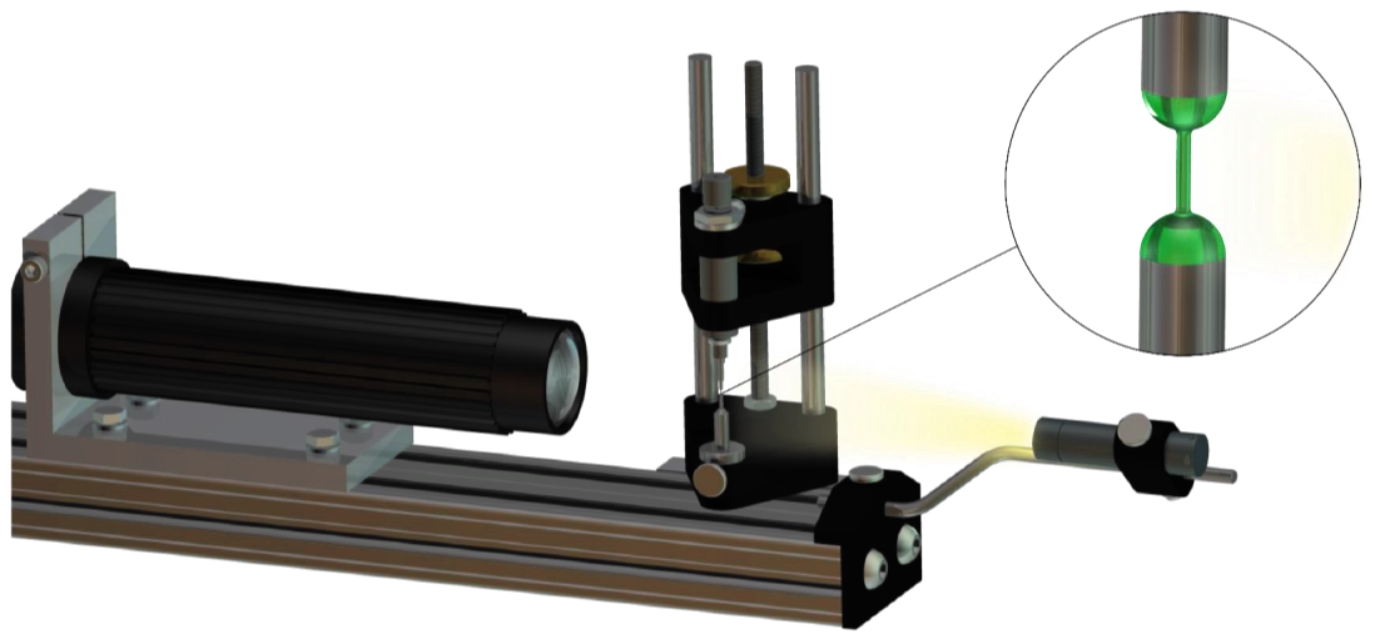

(B)

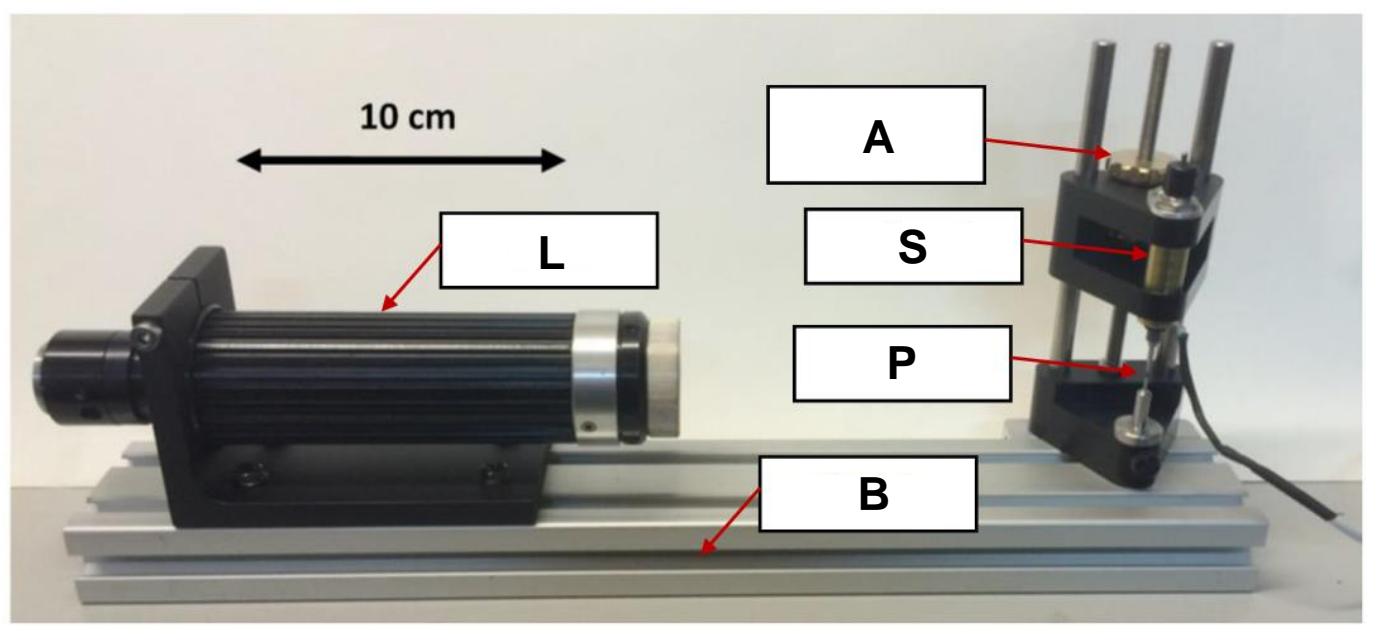

Figure 3. Seymour II (A) Schematic diagram of mounting and mechanism; (B) photograph, with lens (labelled L) attached. B - bracket; P - pistons; S - solenoid; A - adjustment mechanism. 


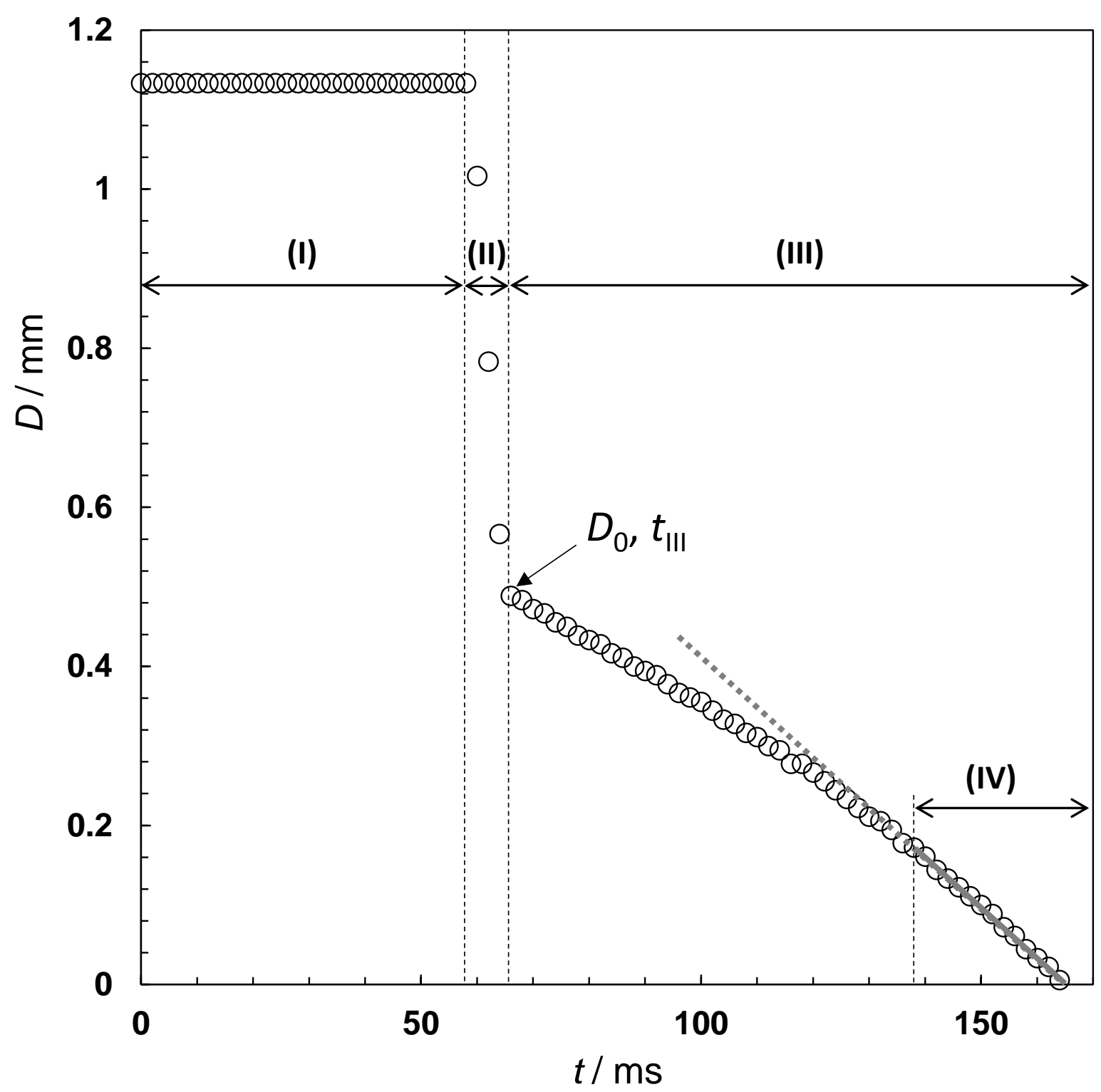

Figure 4. Plot of raw data obtained from Seymour II for a Newtonian fluid (silicone oil). The plot shows a region where the pistons are in their initial position (I), a region where the pistons are moving and a filament of fluid is forming (II) and a region where filament thinning is taking place (III). Also marked is the region where accurate measurement of the Newtonian viscosity can be made ${ }^{[4,30]}$ (IV). 


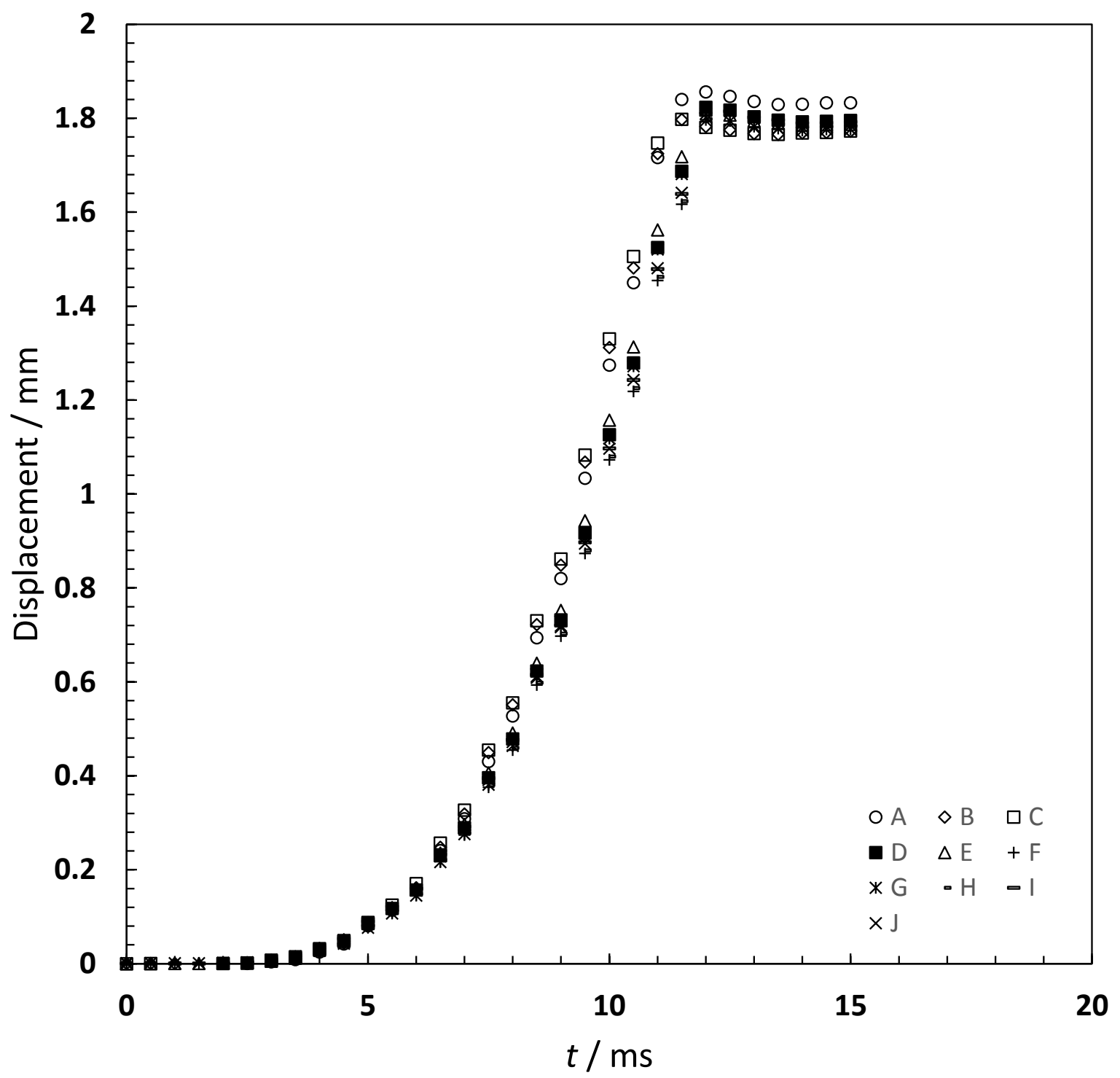

Figure 5. Evolution of piston displacement for ten tests on Seymour II. Gap = $1.8 \mathrm{~mm}$. 


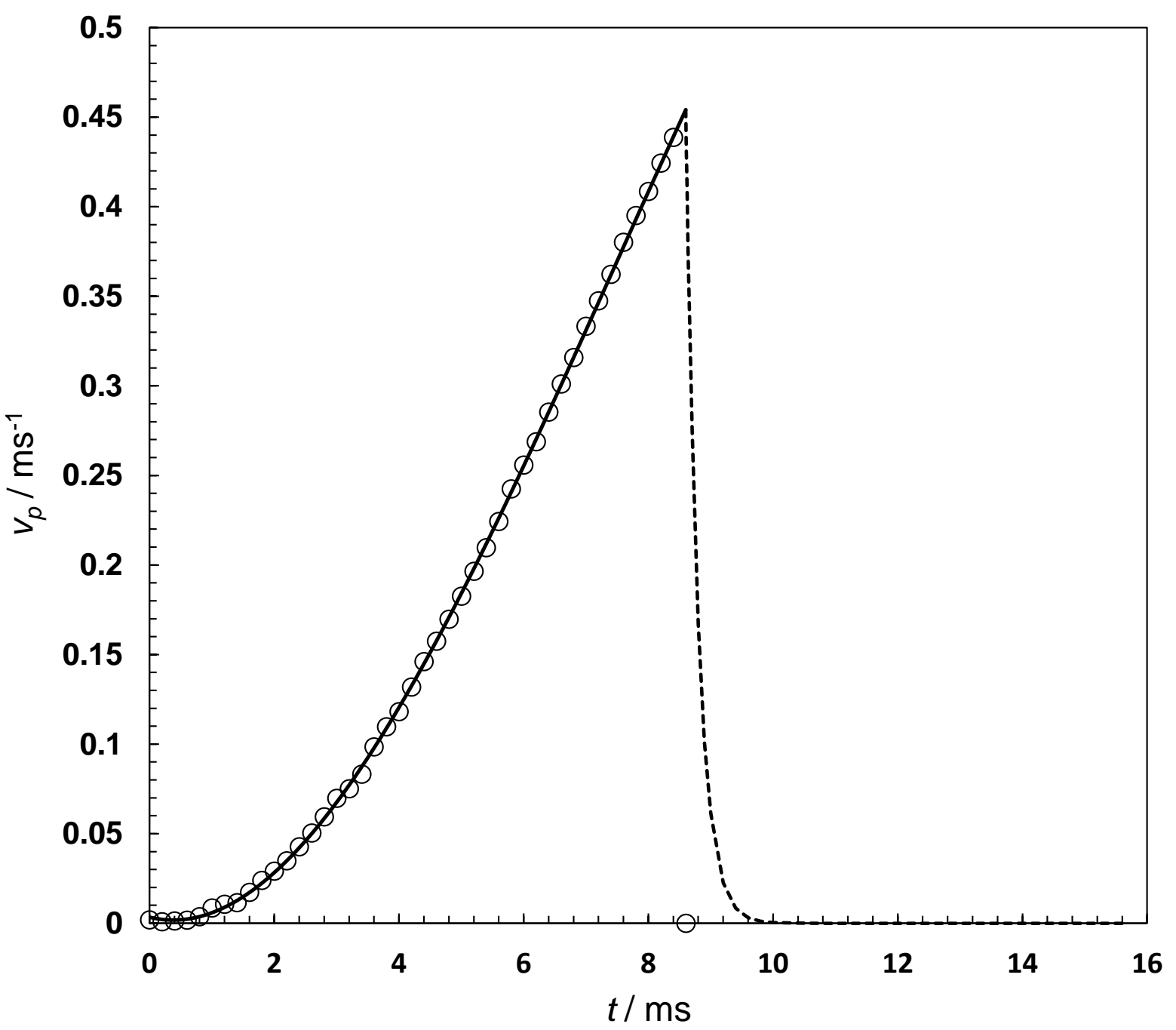

Figure 6. Average piston velocity calculated for data sets in Figure 5 Loci show fitted functions: solid line Equation (1); dashed line - Equation(4). 
Simulation Experiment
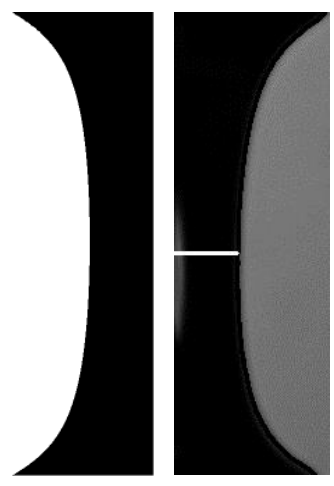

$t=0 \mathrm{~ms}$
Simulation Experiment
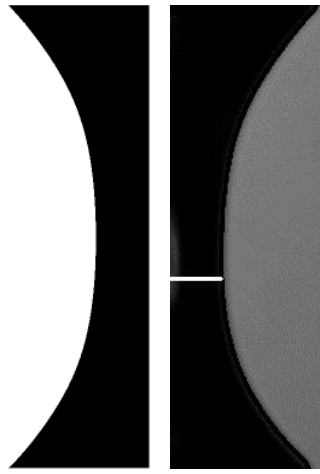

$t=20 \mathrm{~ms}$

Simulation Experiment
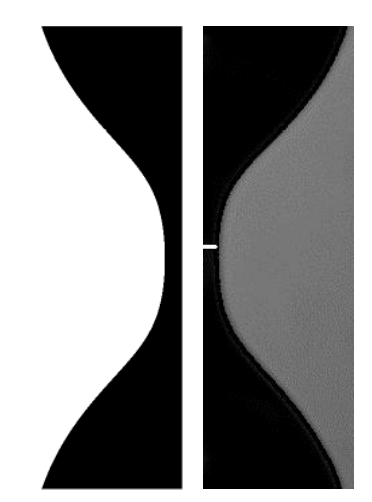

$t=80 \mathrm{~ms}$
Simulation Experiment
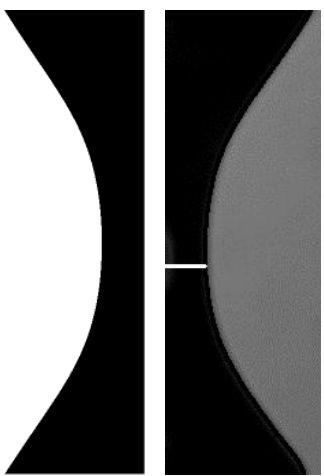

$t=40 \mathrm{~ms}$
Simulation Experiment

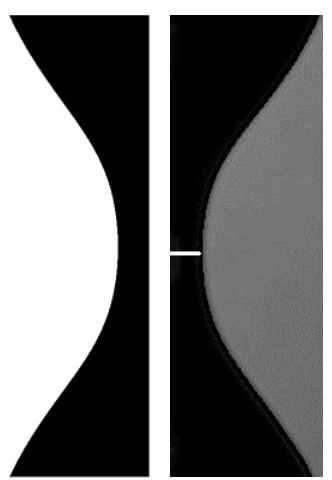

$t=60 \mathrm{~ms}$
Simulation Experiment

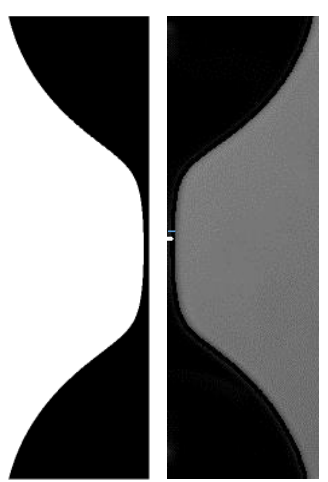

\section{$1 \mathrm{~mm}$}

Figure 7. Comparison of experimental and Polyflow ${ }^{\mathrm{TM}}$ simulation results for a typical filament thinning experiment on Seymour II using $496 \mathrm{mPa}$ s silicone oil. $t$ is time elapsed since pistons stop moving. 


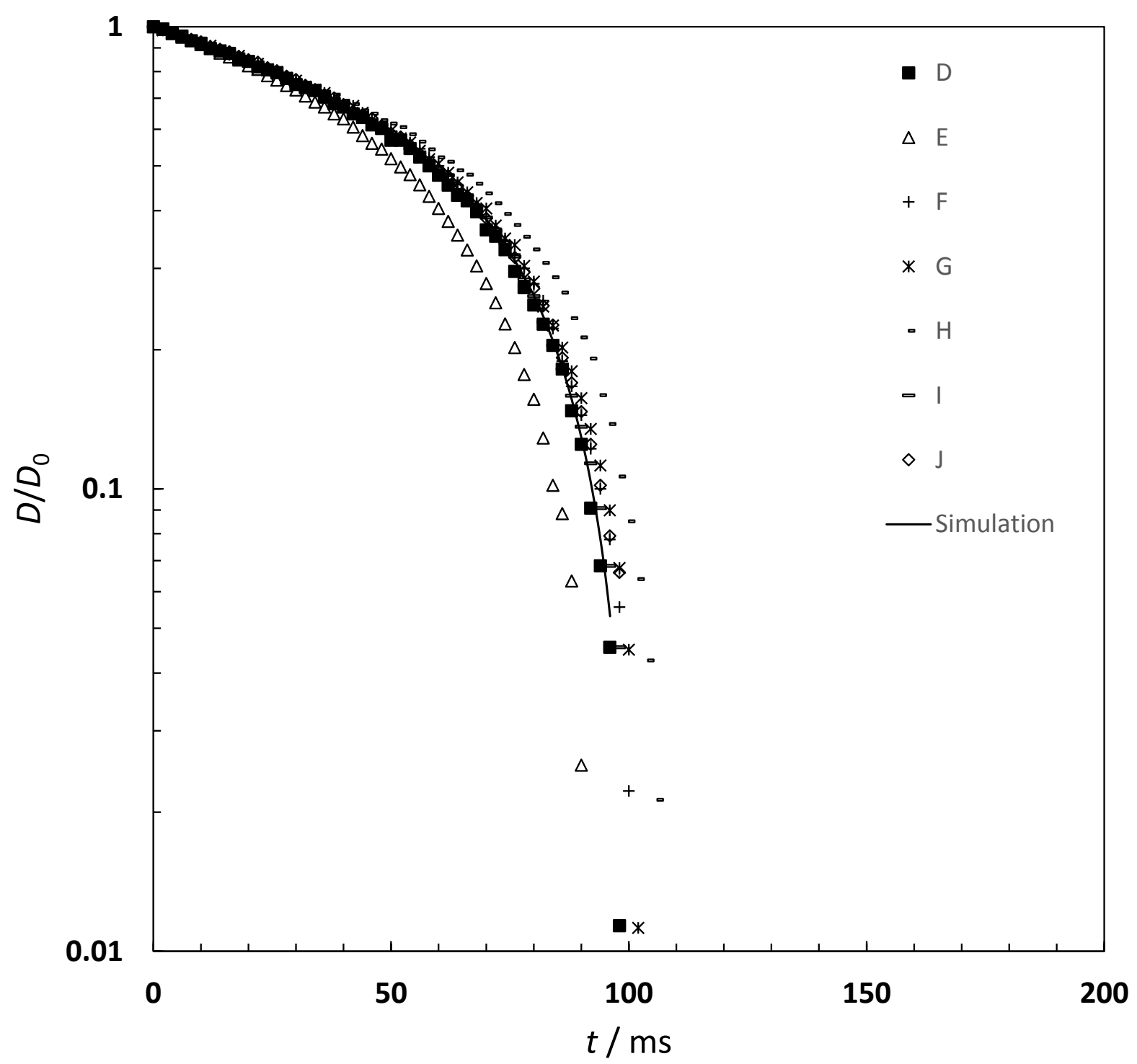

Figure 8. Non-dimensional filament diameter as a function of time for $496 \mathrm{mPa}$ s silicone oil. Points show experimental data collected with Seymour II; locus shows numerical simulation using Polyflow ${ }^{\mathrm{TM}}$. 
(A)

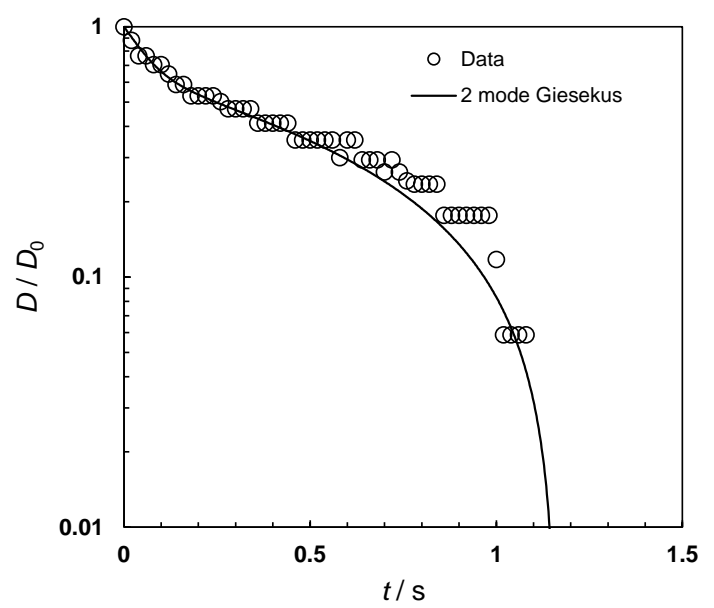

(B)

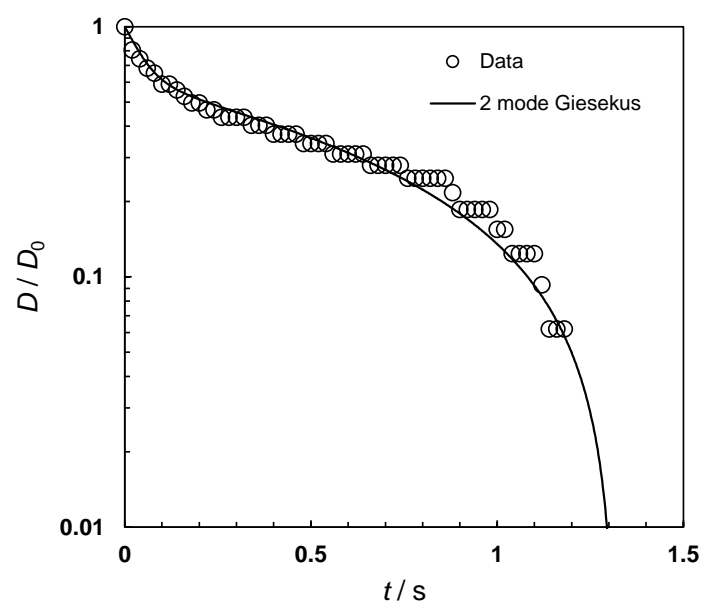

Figure 9. Non-dimensional filament diameter as a function of time for two different samples of mucilage from S. reginae. Points show experimental data collected with Seymour II; loci of points show the solution of Equation (5) using values in Table 4. 


\section{A portable and affordable extensional rheometer for field testing}

Bart Hallmark ${ }^{\dagger}$, Matthew Bryan, Ed Bosson, Simon Butler, Tom Hoier, Ole Magens, Nicolas Pistre*, Lee Pratt, Betsy-Ann Ward, Sam Wibberley and D. Ian Wilson

Department of Chemical Engineering and Biotechnology, New Museums Site, Pembroke Street, Cambridge, CB2 3RA, UK

*École Nationale Supérieure de Techniques Avancées, 828 Boulevard des Maréchaux, 91120 Palaiseau, Paris, France.

†Corresponding author: bh206@cam.ac.uk

\section{SUPPLEMENTARY MATERIAL}

\section{Mesh deformation}

An example of an initial and deformed mesh from the Polyflow ${ }^{\mathrm{TM}}$ simulation is given in Figure S1(A) and Figure S1(B) respectively. The initial mesh, at $t=0 \mathrm{~ms}$, contains 7818 nodes and 7584 elements. Remeshing is carried out automatically by Polyflow ${ }^{\mathrm{TM}}$ when mesh size and quality criteria are exceeded. The final mesh, at $t=94 \mathrm{~ms}$, contains 12998 nodes and 12465 elements.

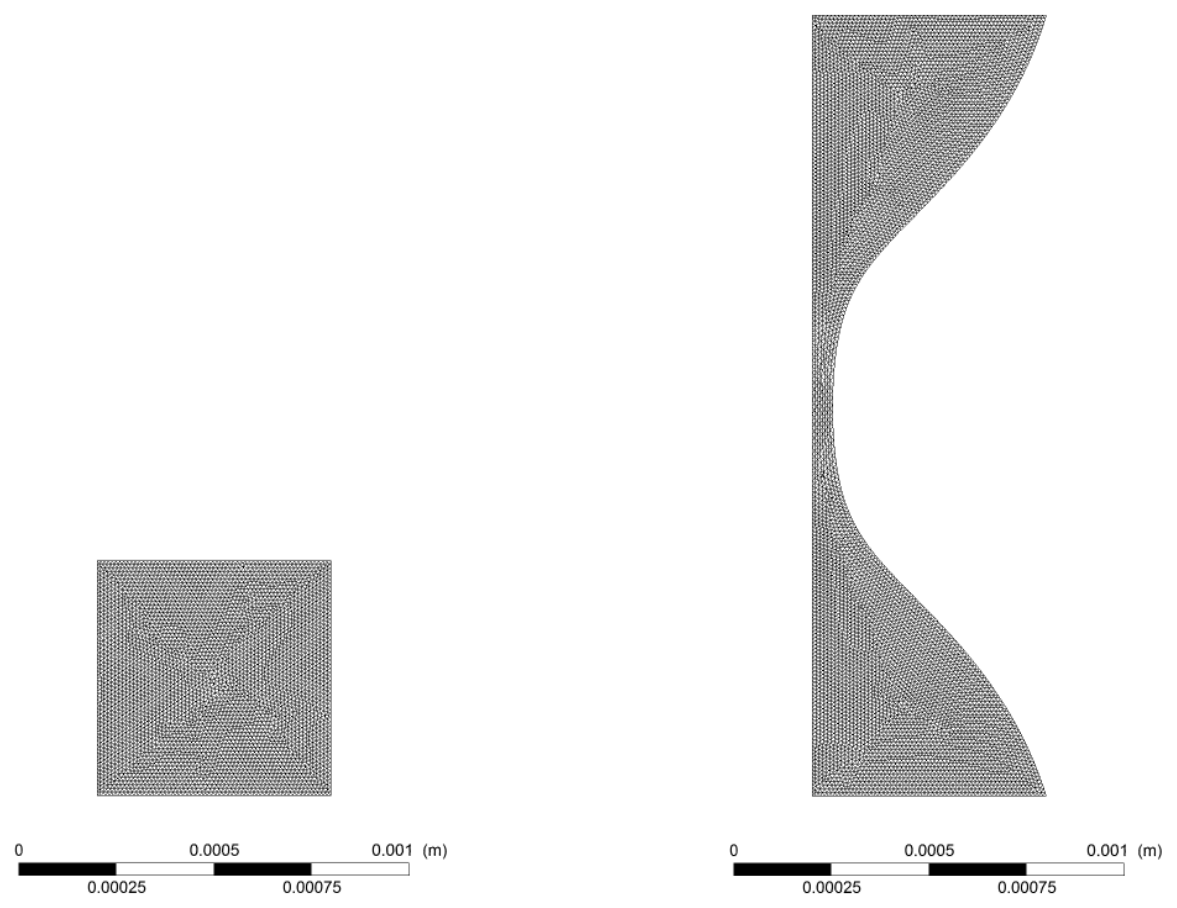

(A)

(B)

Figure S1. Initial (A) and final (B) Polyflow ${ }^{\mathrm{TM}}$ meshes corresponding to simulation times of 0 and $94 \mathrm{~ms}$ respectively. 


\section{CLIPS code for piston movement}

Polyflow ${ }^{\mathrm{TM}}$ has an inbuilt interpreter for the rule-based language CLIPS, as described in the main body of the article. The CLIPS code for the upper piston movement of Seymour is given below.

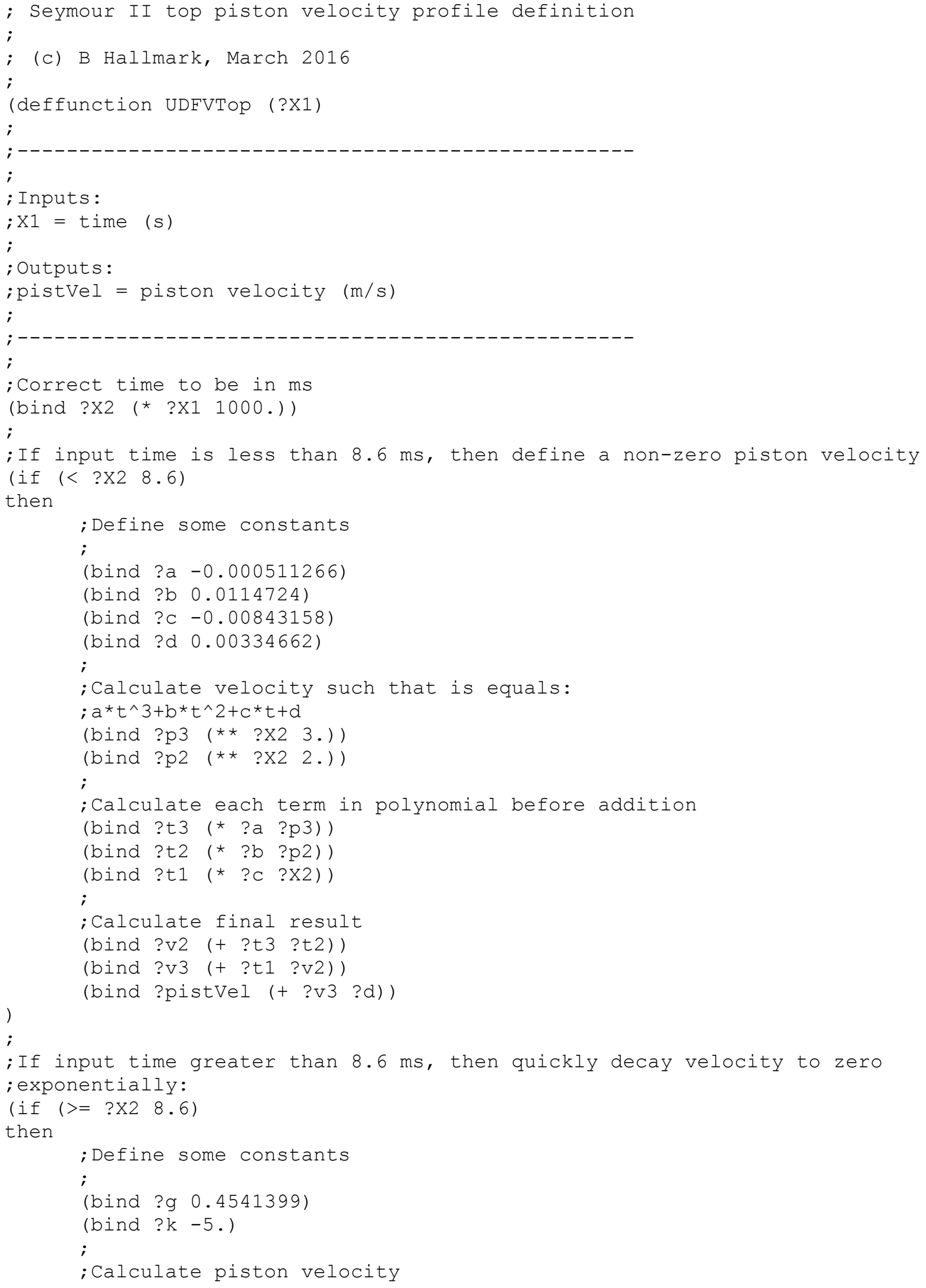




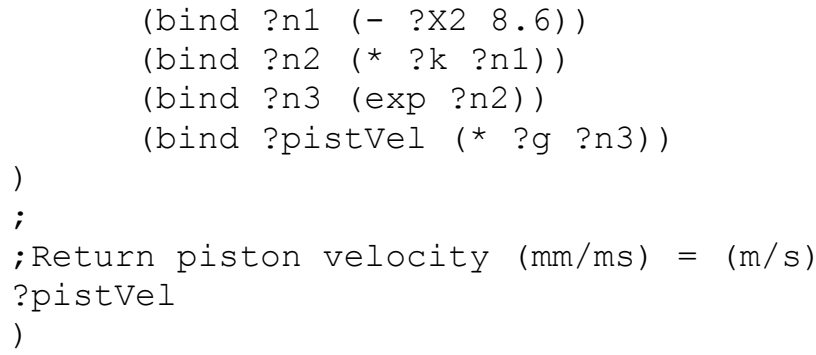

